\title{
Enseñar geografía física en la carrera de turismo a través de un género discursivo del campo profesional
}

\section{Teaching physical geography in tourism through a discursive genre from the professional field}

\author{
Soledad Schwarz ${ }^{1}$, Daniela Stagnaro ${ }^{2}$ \\ sschwarz@untdf.edu.ar,dstagnaro@untdf.edu.ar \\ ${ }^{1}$ Instituto de Desarrollo Económico e Innovación de la Universidad Nacional de Tierra del \\ Fuego, Antártida e Islas del Atlántico Sur (IDEI-UNTDF). Walanika 250, of. 19, Ushuaia. \\ ${ }^{2}$ Instituto de Educación y Conocimiento de la Universidad Nacional de Tierra del Fuego, \\ Antártida e Islas del Atlántico Sur (IEC-UNTDF). Walanika 250, of. 21, Ushuaia.
}

Recibido: 28/05/2020. Aceptado: 14/12/2020

\section{RESUMEN}

A partir de los noventa, en el ámbito de la educación superior en Argentina, se reconoce la importancia que tiene enseñar las formas discursivas propias de las disciplinas científicas durante la formación. En este trabajo, presentamos una propuesta didáctica basada en géneros discursivos para la enseñanza de la materia Geografía Física del primer año de la carrera de Turismo de la Universidad Nacional de Tierra del Fuego, Antártida e Islas del Atlántico Sur. El interés de esta asignatura es que reúne variados campos del conocimiento como geología, climatología, geomorfología y cartografía, y persigue el objetivo de que los estudiantes adquieran herramientas para observar e interpretar los elementos físicos que forman los espacios geográficos en los que la actividad turística se desarrolla.

Aquí, proponemos una estrategia de enseñanza que explota el potencial epistémico y la función social de la escritura para promover la construcción de conocimientos y de identidad profesional dentro de este dominio disciplinar a través de un género específico del turismo: la guía descriptiva en las revistas de viajes. Así, la presente contribución desarrolla una secuencia didáctica centrada en la unidad del programa vinculada al paisaje glacial y se despliega a través de múltiples actividades y desempeños. De esta forma, se busca favorecer la articulación de los contenidos disciplinares en un género discursivo del ámbito profesional para ejercitar la comunicación turística que es un aspecto central en el perfil del egresado de la carrera y fundamental para la actuación en dicho ámbito, pero hasta ahora poco atendido en la formación.

Palabras clave: Geografía Física; Turismo; Alfabetización disciplinar; Géneros profesionales; Secuencia didáctica.

\begin{abstract}
From the nineties, in the field of higher education in Argentina, the importance of learning the discursive forms of scientific disciplines during training is recognized. In this work, we present a didactic proposal for teaching the subject Physical Geography in the first year of a Tourism career at the National University of Tierra del Fuego. The interest of this subject is that it brings together various fields of knowledge such as geology, climatology,
\end{abstract}


geomorphology and cartography, and pursues the objective that students acquire tools to observe and interpret the physical elements that form the geographical spaces in which tourism takes place. Here, we propose a teaching strategy that exploits the epistemic potential and social function of writing to promote the construction of knowledge and professional identity within this disciplinary domain through a specific genre of Tourism: the descriptive guide in travel journals. Thus, the present contribution develops a didactic sequence centred on the unity of the program linked to the glacial landscape and is deployed through multiple activities and performances. In this way, it is sought to favour the articulation of disciplinary contents with a discursive genre of the professional field to exercise tourist communication, a central aspect in the graduate's profile and fundamental for acting in such field, but so far little attended during the student formation.

Keywords: Physical Geography; Tourism; Discipline alphabetization; Professional genres; Didactic sequence.

\section{INTRODUCCIÓN}

"¿Somos coherentes en nuestra modalidad pedagógica con una práctica auténticamente reflexiva?" (Vazquez, 2007, p. 3). Esta pregunta nos interpeló de algún modo como docentes universitarias y nos invitó a pensar estrategias para poner en práctica la escritura como una herramienta para pensar los temas de una asignatura en particular: Geografía Física, materia anual correspondiente al primer año de la carrera de Turismo de la Universidad Nacional de Tierra del Fuego, Antártida e Islas del Atlántico Sur (en adelante, UNTDF).

A través del recorrido que propone esta asignatura, los estudiantes desarrollan herramientas para observar, describir e interpretar los elementos físicos que forman los espacios geográficos en los que la actividad turística se desenvuelve. La interpretación de los procesos formadores del paisaje y su dinámica pasada y actual permite al futuro Guía de Turismo formular discursos coherentes y sostenidos por criterios científicos sobre el recurso que aprovechan. Por otra parte, se brindan herramientas a los futuros Licenciados en Turismo de utilidad para la planificación de la actividad turística sobre un espacio donde se podrá interpretar la dinámica de los procesos formadores y modeladores del relieve en función del clima y el interior de la Tierra. Se trata de una asignatura en la que convergen disciplinas disímiles como la climatología, la geología, la geomorfología y la cartografía, entre otras, de manera que el aprendizaje no es sencillo y mucho menos las múltiples formas discursivas coexistentes.

Una de las estrategias de enseñanza vigente consiste en la lectura tanto de manuales como de textos destinados a especialistas. Los primeros responden a fines didácticos (Cubo de Severino, 2005); los segundos consisten en artículos de revistas científicas, capítulos de libros especializados e informes de investigación. Sin embargo, en ambos casos se busca enfocar, conseguir y motivar la lectura mediante la ayuda de guías para orientar la lectura en función de propósitos y se fomenta la producción de resúmenes propios (Carlino, 2006). No obstante, las prácticas de lectura actuales no se condicen con las prácticas de escritura que los estudiantes tendrán en su futuro profesional. Los estudiantes leen "Geografía Física" pero cuando egresen tendrán que escribir "Turismo". Cassany y Morales (2009) explican:

El aprendizaje de la lectura y la escritura de los textos especializados de cada disciplina es una tarea relevante, que requiere esfuerzo, tiempo y práctica, pues no ocurre de manera natural. El lugar donde se inicia este aprendizaje de 
manera formal es la universidad; sin embargo, no siempre se desarrolla de manera explícita y organizada (p. 109).

Es por ello que la escritura resulta vital para estructurar ideas por escrito según las particularidades de análisis y de organización propias de cada dominio disciplinar y para reescribir las producciones con ojos de lector crítico (Carlino, 2004). De esta forma, en la asignatura objeto de esta presentación, se podría comenzar a explotar el potencial epistémico de la escritura (Miras, 2000; Cassany y Morales, 2009). Más aún, la pregunta inicial de esta introducción hizo evidente la necesidad de equilibrar las prácticas de escritura que los estudiantes llevan a cabo en las instancias prácticas y aquellas que se evalúan en los exámenes parciales. Al respecto, cabe decir que la evaluación cumple distintas funciones: especialmente, sirve para certificar saberes y señalar a los estudiantes qué temas son centrales (Carlino, 2004); sin embargo, interesa reforzar esta instancia como una retroalimentación del aprendizaje y la enseñanza, articulando mejor con el perfil del egresado, sobre todo teniendo en cuenta la importancia que reviste enseñar la comunicación de la comunidad disciplinar del ámbito del turismo, aspecto central en las incumbencias y fundamental para la actuación profesional, pero hasta ahora poco atendido en la formación.

En este marco sociocultural del lenguaje, y desde la perspectiva de los desarrollos actuales sobre las literacidades disciplinares y la didáctica de la escritura basada en géneros discursivos -que serán profundizadas en el acápite siguiente-, esta contribución tiene como objetivo presentar una propuesta de enseñanza que explota el potencial epistémico y la función social de la escritura para promover la construcción de conocimientos y de identidad profesional a través de un género específico del ámbito del turismo: la guía descriptiva en las revistas de viajes. Para ello, en el tercer acápite, se propone una secuencia didáctica centrada en la unidad del programa de la materia vinculada al paisaje glacial que implica el despliegue de múltiples actividades y desempeños. Asimismo, la propuesta revisa particularmente las formas de evaluación vigentes, reconociendo que "cambiar las prácticas evaluativas usuales para acercarse a las deseables no depende sólo de la voluntad individual del docente, sino también de la cultura académica institucional en la que se desempeña" (Carlino, 2004, p. 8) pero entendiendo que el primer paso consiste en -al menos- pensar cómo intentar modificarlas.

\section{PERSPECTIVAS TEÓRICAS}

\subsection{Literacidades disciplinares}

Esta propuesta se inscribe en los incipientes desarrollos de lo que se denomina literacidades disciplinares que hace foco en las particularidades de las prácticas de cada disciplina (Lillis y Scott, 2007; Lea y Street, 2006). Desde esta perspectiva, se atiende tanto a los procesos de enseñanza y de aprendizaje como a las herramientas y los conocimientos necesarios para que los estudiantes logren acceder a las prácticas disciplinares para producir y transformar los conocimientos (Moje, 2007).

Así, el interés de la enseñanza no se limita exclusivamente a la lectura y la escritura, sino que propende a motorizar la participación de los estudiantes en actividades similares a las desempeñadas por los profesionales de la comunidad disciplinar con el propósito de promover aprendizajes de los conocimientos y epistemologías propias de la disciplina y prepararlos para las prácticas profesionales (Carlino, 2013; López-Bonilla, 2013; Moje, 2007; Ford y Forman, 2006). 
Entendiendo los géneros discursivos como formas relativamente estables específicas de cada esfera o ámbito de la actividad humana (Bajtín, 2008 [1979]) con funciones cognitivas, interpersonales y sociopolíticas (Cassany, 2008; Cassany y Morales, 2009), inscribimos el trabajo en las actuales propuestas de la didáctica de la escritura basada en géneros discursivos (Natale y Stagnaro, 2013; Navarro, 2019). Esta categoría resulta particularmente potente en términos didácticos en tanto contribuye a promover aprendizajes significativos desde una perspectiva socioconstructivista y crítica que articula contextos de producción culturales y situacionales con los propósitos comunicativos y el sistema de opciones lingüísticas entre las que elige el hablante/escritor. A su vez, buscamos acortar la brecha entre las prácticas letradas del ámbito de formación y el profesional evidenciada por Cassany y López (2010) vinculando de manera directa las propuestas de escritura con los alcances y capacidades profesionales que establecen los planes de estudio de las carreras. De esta manera, se apunta a formar al estudiante como miembro de una comunidad disciplinar desde cada una de las materias partiendo de las demandas discursivas propias de la práctica profesional.

\subsection{Los géneros discursivos del turismo}

Los géneros discursivos dentro de cada esfera de la actividad humana se organizan en función de objetivos sociales y comunicativos. Así, en cada esfera social, se manejan géneros que abordan determinados temas a partir de formas de estructuración particulares y estilos lingüísticos propios del ámbito.

En el campo disciplinar del turismo se producen, circulan y consumen géneros discursivos relativos a esta actividad. Al respecto cabe mencionar el aporte de Calvi (2010) quien plantea que las prácticas sociales y las correspondientes producciones textuales pueden agruparse en tres bloques: por un lado, existen los géneros para la reflexión teórica sobre el fenómeno turístico; por el otro, los géneros propios de la gestión del turismo (como por ejemplo, el contrato de viaje); y por último, aquellos utilizados para la descripción y promoción de los lugares turísticos (como guías y folletos). Considerando los objetivos de la asignatura, es este tercer bloque el que nos permite pensar al turismo como medio de difusión de las características de un territorio ya que emplea diversos géneros para describir y promocionar los lugares turísticos, incluido su patrimonio natural. Calvi (2010) clasifica los géneros del turismo en familias de géneros tales como editoriales - guías de viajes y revistas de viajes-, institucionales -folletos, anuncios de destinos turísticos, webs institucionales-, comerciales anuncios comerciales, catálogos de viajes, folletos de servicios turísticos-, organizativos billetes, reservas, cartas, facturas, contratos-, legales -normativas, reglamentos-, científicos y académicos -artículos y libros- e informales -foros y blogs de viajeros-. Por otro lado, la autora identifica macrogéneros como guías de turismo, folletos, revistas de viaje, catálogos de viajes y páginas web, dentro de los cuales distingue guías descriptivas, itinerarios, guías prácticas, programas de viaje, reportajes, anuncios, informes, etc.

A partir de esta clasificación, en esta propuesta se seleccionó el género guía descriptiva dentro del macrogénero revista de viaje.

\section{PROPUESTA DE ENSEÑANZA}

Como se ha planteado en la introducción, la presente secuencia didáctica tiene como objetivo generar instancias de escritura coherentes con los géneros propios del turismo así como propiciar un correlato efectivo entre lo que se lee y escribe durante la cursada y los modos de evaluación, atendiendo a las competencias del futuro egresado. Para ello, se eligió la unidad 4 
titulada "Paisaje glacial" del módulo 4 "Procesos exógenos" de la asignatura Geografía Física, que corresponde al segundo cuatrimestre del primer año de la carrera.

Los contenidos mínimos de esta unidad son: nieve, neviza y hielo glaciario; glaciares: definición, clasificación, dinámica, distribución; línea de nieve; geoformas de erosión y acumulación; evolución del paisaje glacial; glaciaciones en Tierra del Fuego y Patagonia.

El objetivo general de la unidad consiste en que el estudiante desarrolle una visión integradora de los procesos de génesis glacial que se producen en el paisaje, internalizando el concepto de paisajes dinámicos. De allí se desagregan los siguientes objetivos específicos: identificar los elementos y procesos de génesis glacial existentes en un determinado espacio geográfico; interpretar paisajes glaciales a partir del uso de fotografías; conocer los eventos glaciales recientes que afectaron Patagonia en general y Tierra del Fuego en particular; producir una breve guía descriptiva de revista de viajes.

A partir de la secuencia didáctica que se desplegará más adelante se pretende que los estudiantes logren observar, relacionar y aplicar conceptos a partir de la interpretación y análisis de textos, tablas, gráficos, esquemas, mapas y fotografías; asimismo, se fomenta que puedan exponer y relacionar conceptos ante un pequeño grupo, trabajando responsablemente en equipo y ensayando el trabajo autónomo; también se busca propiciar un acercamiento a las prácticas profesionales a partir de la producción del género guía descriptiva de revista de viajes propio de este ámbito. La propuesta de reelaboración de los contenidos de la asignatura a partir del trabajo con este género profesional busca promover aprendizajes significativos a la vez que favorecer el desarrollo de identidades profesionales a partir de situaciones retóricas que proponen al escritor universitario posicionarse como profesional del turismo en sus textos, es decir, se apunta al trabajo tanto con la función epistémica como con la interpersonal y la sociopolítica (Cassany, 2008).

Con respecto a las prácticas de lectura que se promueven, la propuesta, atendiendo a los diagnósticos y aportes de las investigaciones (por ejemplo, di Stefano y Pereira, 2009), busca guiar y acompañar a los estudiantes en la lectura académica y lo que se espera de ellas en la materia. Para ello, en relación con la unidad que aquí nos ocupa se ofrecen los siguientes recursos didácticos:

- Un cuadernillo de apuntes (en adelante, CA) diseñado por el equipo de cátedra que recupera y enfatiza los principales contenidos a partir de las fuentes bibliográficas correspondientes. Este recurso se facilita en forma digital a los estudiantes permitiéndoles imprimirlo para favorecer la toma de apuntes en clase.

- Una presentación en formato PowerPoint (en adelante, PPT) basada en el CA. En la Figura 1 se pueden consultar algunas diapositivas a modo de ejemplo.

- Materiales de lectura general (en adelante, MLG) compuestos por géneros didácticos que apuntan a introducir a los estudiantes en tanto lectores no expertos en las temáticas abordadas y materiales de lectura específica (en adelante, MLE) que se indican con posterioridad con el objeto de graduar la especialidad de los géneros de las lecturas que hacen los estudiantes. De acuerdo con la clasificación propuesta por Cubo de Severino (2005), los MLG consisten en capítulos de manuales académicos -que además incluyen glosarios-, y los MLE están conformados por artículos científicos y de divulgación. 


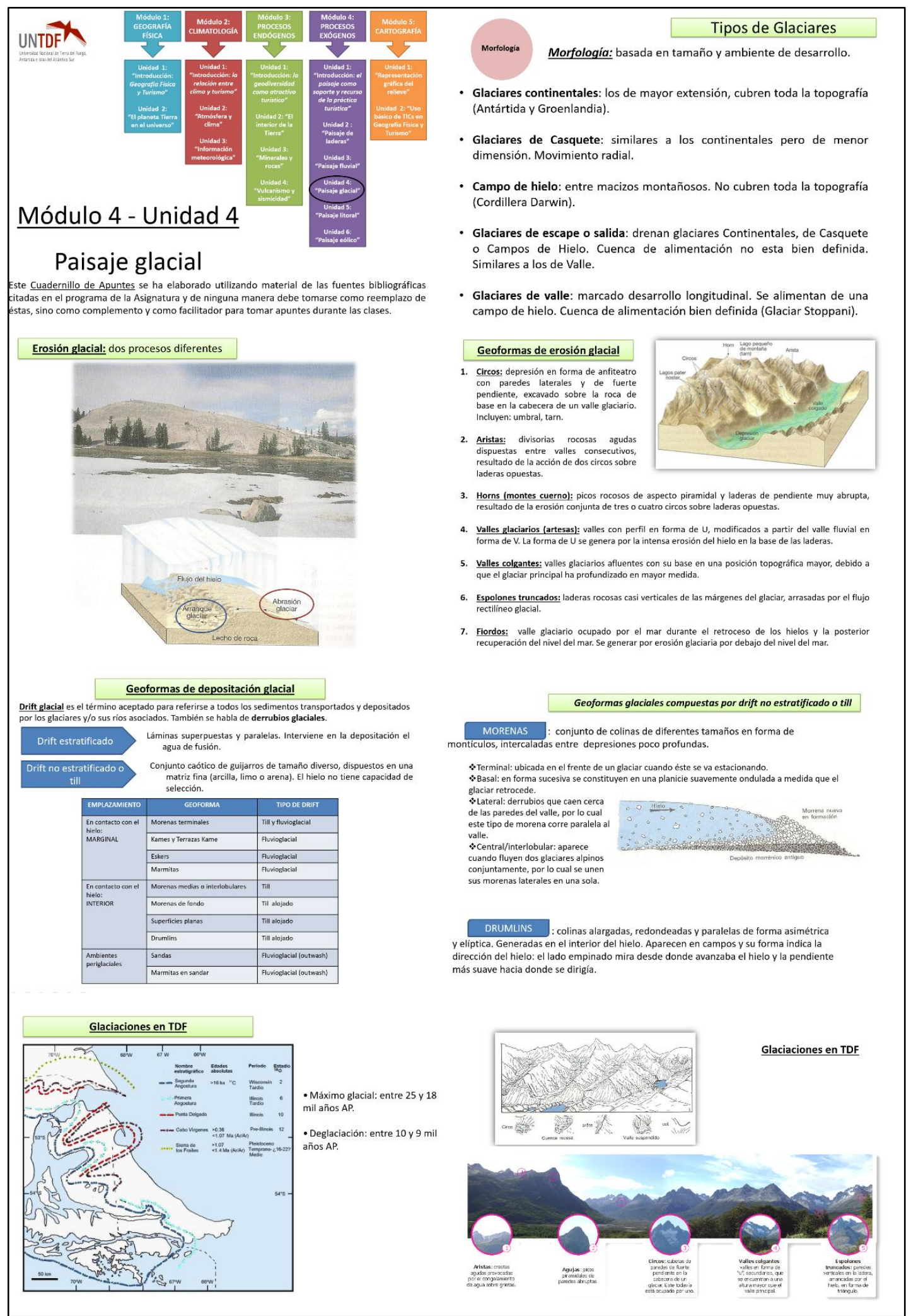

Figura 1. Extracto del CA.

Fuente y elaboración del equipo de cátedra de Geografía Física (IDEI/UNTDF).

- Una guía de lectura y ejercicios (en adelante, GLE) diseñada por el equipo de cátedra para que los estudiantes completen en forma áulica y/o domiciliaria, en pequeños grupos y/o en forma individual. Esta guía se diseñó con el propósito de orientar a los estudiantes en la lectura en función de los objetivos de la asignatura y contribuir a que puedan abordar con más 
eficiencia los MLG y los MLE (Carlino, 2006). Además, consta de varias partes que buscan promover el desarrollo de diversos desempeños por parte de los estudiantes. En la Figura 2 pueden observarse algunas de las consignas elaboradas.

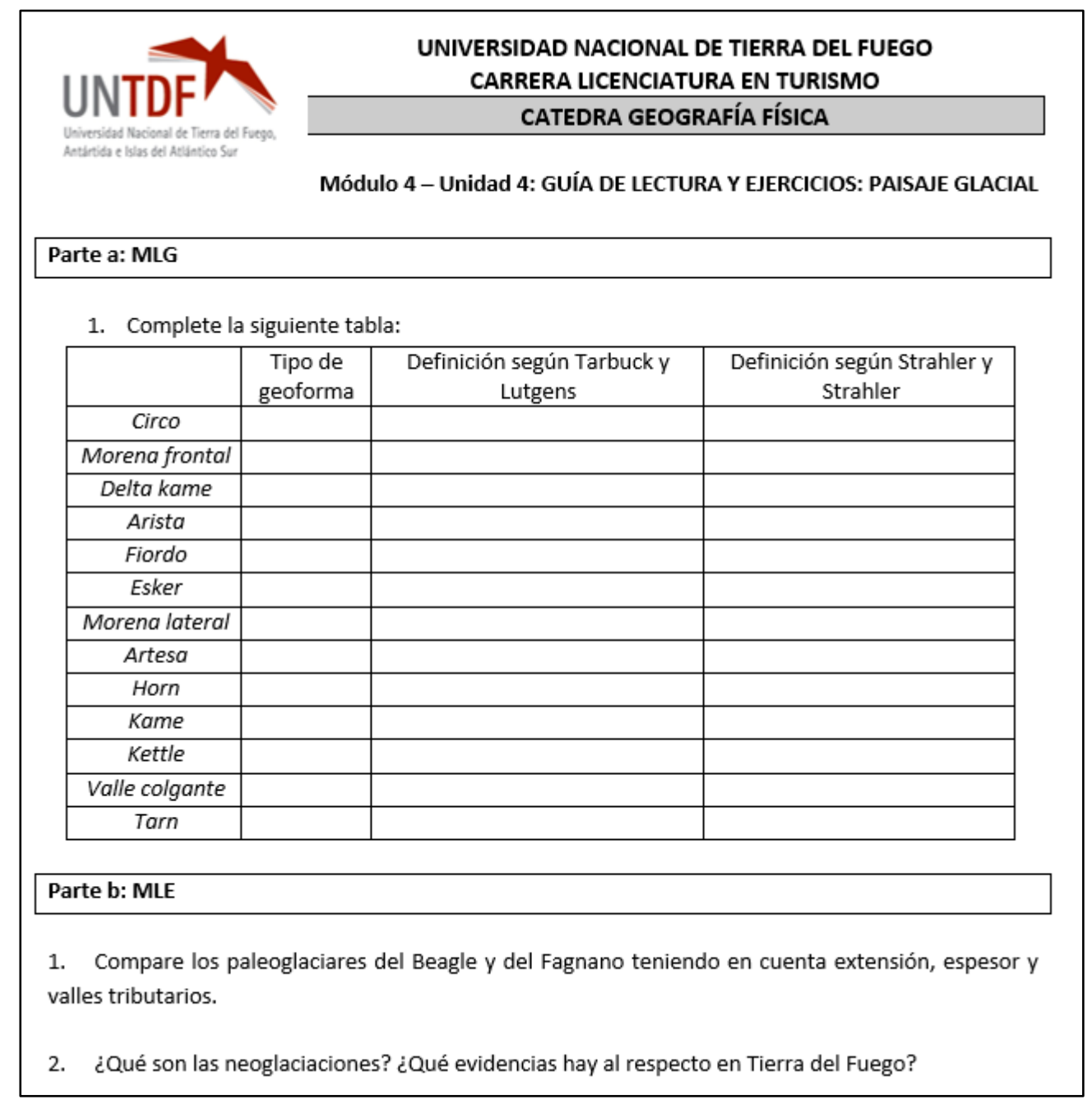

Figura 2. Extracto de la GLE.

Fuente y elaboración del equipo de cátedra de Geografía Física (IDEI/UNTDF).

- Un ejemplar genérico de guía descriptiva dentro de una revista de viajes (en adelante, GD) para que los estudiantes cuenten con una realización del género a producir que se analiza de forma conjunta (Moyano, 2003) para identificar las distintas partes y funciones del texto, y los usos del lenguaje especializado. La GD se presenta como una oportunidad para aprender una forma de lectura y escritura especializadas dentro del Turismo ya que, como plantean Cassany y Morales (2009), este aprendizaje debe iniciarse dentro de la universidad y no una vez concluida esta.

- Un trabajo evaluativo final de la unidad (en adelante, TEFU) que funciona como instancia de evaluación integradora (Figura 3). Consiste en un trabajo de redacción individual de un ejemplar del género guía descriptiva, de acuerdo con la clasificación propuesta por Calvi (2010). Siguiendo a Carlino (2004), con el TEFU apuntamos a una evaluación deseable, ya que está formulado en correlato con las prácticas de lectura y escritura llevadas a cabo durante el desarrollo de la unidad y, además, enfocado en las producciones discursivas propias del ámbito profesional de desempeño del futuro egresado. La evaluación es acompañada por una lista de cotejo (Tabla 1) que sirve de herramienta al estudiante en las distintas etapas 
recursivas del proceso de escritura: planificación, puesta en texto y revisión (Hayes y Flower, 1981), a la vez que facilita la retroalimentación (Anijovich, 2010) con el estudiante.

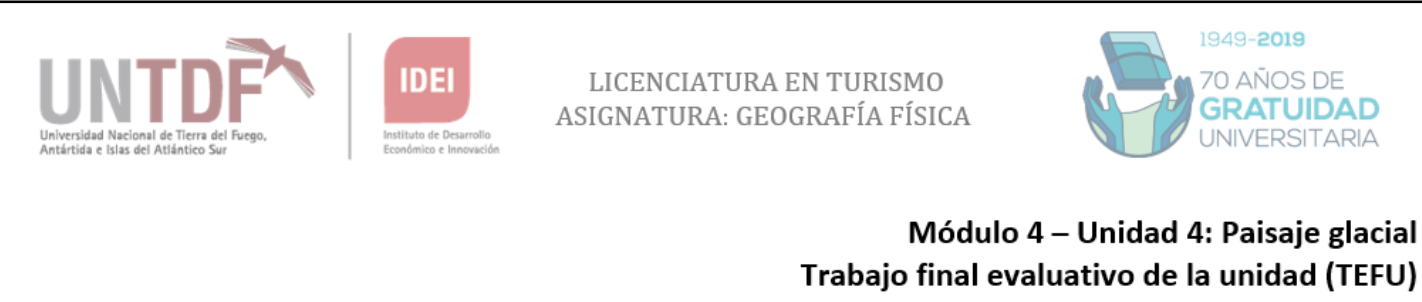

CONSIGNA

A partir de la imagen seleccionada, los contenidos teóricos tanto generales como específicos y el modelo de texto correspondiente a una guía descriptiva dentro de una revista de viajes y turismo, describa el paisaje observado apelando a la interpretación de los procesos de génesis glacial que modelaron la superficie; la narración debe ser breve (hasta mil palabras) y debe incluir vocabulario técnico.

\section{EJERCICIOS}

1) Identifique geoformas de erosión y depositación glacial en la imagen.

2) Planifique la descripción haciendo uso de la bibliografía de la unidad.

3) Redacte el primer borrador.

4) Lea en su pequeño grupo la narración para que sus compañeros descubran qué imagen está describiendo.

5) Ajuste su borrador y entregue.

Figura 3. TEFU.

Fuente y elaboración propias.

Tabla 1. Lista de cotejo.

\begin{tabular}{|l|l|l|}
\hline \multicolumn{2}{|c|}{ Categorías } & Observaciones \\
\hline La interpretación es pertinente. & & \\
\hline $\begin{array}{l}\text { La información está claramente organizada y } \\
\text { es completa. }\end{array}$ & & \\
\hline $\begin{array}{l}\text { El vocabulario técnico es utilizado } \\
\text { correctamente. }\end{array}$ & & \\
\hline $\begin{array}{l}\text { No hay errores de gramática, ortografía o } \\
\text { puntuación. }\end{array}$ & & \\
La extensión del texto es la solicitada. & & \\
\hline
\end{tabular}

Fuente y elaboración propias.

De esta manera, los distintos recursos didácticos se articulan en el desarrollo de las clases en función de los objetivos de la asignatura promoviendo el trabajo guiado con distintos géneros discursivos propios de diferentes ámbitos: por ejemplo, el CA y el PPT responden al contexto universitario, los MLG y MLE dan cuenta de las formas específicas dentro de la disciplina Geografía Física mientras que la GD promueve las literacidades propias del Turismo, tal como veremos más adelante en el esquema de secuencia didáctica.

En la Tabla 2 se presenta la secuencia completa que involucra al menos diez clases teóricoprácticas. Tal como podrá leerse en la misma, parte de las actividades a realizar por los estudiantes se vertebran en un proceso que, en términos de Flower y Hayer (1981), implica planificar la escritura, realizar la puesta en texto y revisar la producción -lo cual es propuesto, 
tal como lo sugieren Dolz y Schneuwly (2011) mediante la discusión en grupos o pares-, siempre reflexionando en torno al problema retórico a medida que se va produciendo el texto.

Siguiendo a Cassany y López (2010), esta secuencia busca el vínculo entre las prácticas letradas -mediante la guía de lectura y ejercicios así como a través del trabajo evaluativo final de la unidad- y el perfil profesional propuesto por plan de estudio como un modo de inmersión del estudiante en las prácticas propias de la comunidad disciplinar en la que aspira a ingresar como miembro. Así, la propuesta atiende a una demanda discursiva propia del Turismo como disciplina al mismo tiempo que introduce al estudiante dentro de una comunidad académica específica.

Tabla 2. Propuesta de secuencia didáctica

\begin{tabular}{|c|c|c|}
\hline Contenidos & Actividades secuenciadas & Recursos \\
\hline $\begin{array}{l}\text { Nieve, neviza, } \\
\text { hielo glaciario. } \\
\text { Clasificación de } \\
\text { glaciares. Línea } \\
\text { de nieve. } \\
\text { (1 clase) }\end{array}$ & $\begin{array}{l}\text { - Los docentes presentan los contenidos mediante el PPT para } \\
\text { que los estudiantes sigan su CA. } \\
\text { - Se chequea comprensión mediante preguntas-control orales. } \\
\text { - Los docentes presentan el TEFU. } \\
\text { - Se indica a los estudiantes realizar lectura domiciliaria de } \\
\text { MLG. }\end{array}$ & $\begin{array}{ll}\text { - } & \text { CA } \\
\text { - } & \text { PPT } \\
\text { - } & \text { MEFU } \\
\text { MLG }\end{array}$ \\
\hline $\begin{array}{l}\text { Geoformas de } \\
\text { erosión y de } \\
\text { depositación. } \\
\text { Evolución del } \\
\text { paisaje glacial. (1 } \\
\text { clase })\end{array}$ & $\begin{array}{l}\text { - Los docentes presentan los contenidos mediante el PPT para } \\
\text { que los estudiantes sigan su CA. } \\
\text { - Los estudiantes realizan lectura individual de los manuales } \\
\text { académicos indicados en el MLG. } \\
\text { - Los docentes presentan la GLE. } \\
\text { - Los estudiantes completan la parte A de la GLE en pequeños } \\
\text { grupos. }\end{array}$ & $\begin{array}{l}\text { - } \text { CA } \\
\text { - } \text { PPT } \\
\text { - } \text { GLE, parte A } \\
\text { - } \text { MLG }\end{array}$ \\
\hline $\begin{array}{l}\text { Guía descriptiva } \\
\text { en revista de } \\
\text { viajes } \\
(1 \text { clase })\end{array}$ & $\begin{array}{l}\text { - Los docentes presentan una GD indicando sus partes y } \\
\text { especificidades. } \\
\text { - Los estudiantes completan el primer ejercicio del TEFU. } \\
\text { - Se indica a los estudiantes realizar lectura domiciliaria de } \\
\text { MLE. }\end{array}$ & $\begin{array}{ll}\text { - } & \text { GD } \\
\text { - } & \text { TEFU } \\
\text { - } & \text { MLE }\end{array}$ \\
\hline $\begin{array}{l}\text { Glaciaciones en } \\
\text { Patagonia y } \\
\text { Tierra del Fuego } \\
\quad(2 \text { clases })\end{array}$ & $\begin{array}{l}\text { - Un docente invitado, experto en el tema, presenta contenidos } \\
\text { mediante PPT. } \\
\text { - Los estudiantes realizan preguntas en función de la } \\
\text { presentación y del MLE abordado. } \\
\text { - Los estudiantes realizan lectura individual áulica de MLE. } \\
\text { - Se debate la lectura en pequeños grupos. } \\
\text { - Los estudiantes completan la parte B de la GLE. } \\
\text { - Los estudiantes completan el segundo ejercicio del TEFU con } \\
\text { la guía del equipo docente. }\end{array}$ & $\begin{array}{l}\text { - Presentación PPT } \\
\text { del docente invitado } \\
\text { - MLE } \\
\text { - GLE, parte B. } \\
\text { - TEFU }\end{array}$ \\
\hline $\begin{array}{l}\text { Toda la unidad }(2 \\
\text { clases })\end{array}$ & $\begin{array}{l}\text { - Puesta en común de la GLE mediante exposición por parte de } \\
\text { los estudiantes. } \\
\text { - Los estudiantes comienzan a completar el tercer ejercicio del } \\
\text { TEFU y lo finalizan en forma domiciliaria. }\end{array}$ & $\begin{array}{l}\text { - } \text { GLE } \\
\text { TEFU }\end{array}$ \\
\hline $\begin{array}{c}\text { TEFU } \\
(2 \text { clases })\end{array}$ & $\begin{array}{l}\text { - Los estudiantes realizan el cuarto ejercicio del TEFU en } \\
\text { pequeños grupos. } \\
\text { - Los estudiantes completan el último ejercicio del TEFU y } \\
\text { entregan. }\end{array}$ & - TEFU \\
\hline $\begin{array}{c}\text { TEFU } \\
(1 \text { clase })\end{array}$ & $\begin{array}{l}\text { - Los docentes realizan la devolución del TEFU en forma } \\
\text { individual a cada estudiante. }\end{array}$ & - TEFU \\
\hline
\end{tabular}

Fuente y elaboración propias.

\section{COMENTARIOS FINALES}

Enseñar Geografía Física en la carrera de Turismo de la UNTDF exige comprender cómo se entrama la materia en el plan de estudios y su relación con los alcances del título. En ese 
sentido, Cassany (2003) sostiene que los textos no son formatos universales ni neutros, sino que están situados en una comunidad disciplinar particular que impone sus formas de producción e interpretación, por lo que es necesaria en la formación la enseñanza de las formas de comunicación propias de esa comunidad disciplinar, es decir que "el sujeto debe aprender los usos particulares (géneros, prácticas culturales, tradición)" (p. 42) de la comunidad en la que se inscribe cada carrera. Por lo tanto, vimos que también es necesario enseñar las formas particulares en que los contenidos de tal asignatura se comunican en el campo de actuación laboral del futuro egresado de Turismo.

En línea con lo anterior, hemos presentado una propuesta didáctica que apunta a tender puentes entre el ámbito de formación y el ámbito profesional a partir de la articulación de contenidos disciplinares en un género profesional vinculado con los alcances del título y el perfil de egresado que propone la carrera.

Si bien la propuesta desplegada no se ha implementado aún, consideramos que -en línea con los supuestos teóricos presentados- posee una elevada potencialidad para explotar la función epistémica de la escritura y para generar identidad profesional. La inminente puesta en acción de la secuencia en el próximo año académico y su posterior análisis nos permitirá comunicar los resultados de esta estrategia ligada a la literacidad disciplinar a través de un género discursivo del ámbito profesional del turismo en futuros trabajos.

\section{REFERENCIAS BIBLIOGRÁFICAS}

ANIJOVICH, R. (comp.) (2010). La evaluación significativa. Buenos Aires: Paidós.

BAJTÍN, M. (2008[1979]). El problema de los géneros discursivos. En Estética de la creación verbal. Buenos Aires: Siglo Veintiuno Editores.

CALVI, M. (2010). Los géneros discursivos en la lengua del turismo: una propuesta de clasificación. Ibérica, 19: 9-32.

CARLINO, P. (2004). La distancia que separa la evaluación escrita frecuente de la deseable. Acción Pedagógica, 13(1): 8-17.

CARLINO, P. (2006). Ayudar a leer en los primeros años de universidad o de cómo convertir una asignatura en «materia de cabecera». Educación en Ciencias: 1-12.

CARLINO, P. (2013). Alfabetización académica diez años después. Revista Mexicana de Investigación Educativa, 355-381.

CASSANY, D. (2003). La lectura y la escritura de géneros profesionales en EpFE. Actas del Segundo Congreso Internacional de Español para Fines Específicos. Ámsterdam, noviembre 2003.

CASSANY, D. (2008). Géneros escritos. En Taller de textos. Leer, escribir y comentar en el aula (pp. 17-48). Buenos Aires: Paidós.

CASSANY, D. y LÓPEZ, C. (2010). De la universidad al mundo laboral: continuidad y contraste entre las prácticas letradas académicas y profesionales. En G. Parodi, Alfabetización académica y profesional en el siglo XXI. Leer y escribir desde las disciplinas (pp. 347-374). Chile: Planeta.

CASSANY, D. y MORALES, O. A. (2009). Leer y escribir en la universidad: los géneros científicos. En D. Cassany (comp.), Para ser letrados. Voces y miradas sobre la lectura (109-128). Barcelona, Paidós.

CUBO DE SEVERINO, L. (2005). Los manuales universitarios. En Los textos de la ciencia. Principales clases del discurso académico. Córdoba: Comunicarte.

DI STEFANO, M. y PEREIRA, M. C. (2009). Representaciones sociales en el proceso de lectura. Signo y Seña, 8: 317-340. 
DOLZ, J. y SCHNEUWLY, B. (2011). Escribir es reescribir. La reescritura en las secuencias didácticas para la expresión escrita. Leer.es (Enseñar a escribir. Enseñar a pensar).

FLOWER, L. y HAYES, J. (1981). A cognitive process theory of writing, College Composition and Communication, 32 (4), 365-387.

FORD, M. J. y FORMAN, E. A. (2006). Redefining disciplinary learning in classroom contexts. Review of research in education, 30: 1-32.

LEA, M. R. y STREET, B. V. (2006). The "academic literacies" model: Theory and applications. Theory into practice, 45(4): 368-377.

LILLIS, T. y SCOTT, M. (2007). Defining academic literacies research: Issues of epistemology, ideology and strategy. Journal of Applied Linguistics, 4(1): 5-32.

LÓPEZ-BONILLA, G. (2013). Prácticas disciplinares, prácticas escolares: Qué son las disciplinas académicas y cómo se relacionan con la educación formal en las ciencias y en las humanidades. Revista Mexicana de Investigación Educativa, 18(57): 383-412.

MIRAS, M. (2000). La escritura reflexiva. Aprender a escribir y aprender acerca de lo que se escribe. Infancia y Aprendizaje, 89: 65-80

MOJE, E. B. (2007). Developing socially just subject-matter instruction: A review of the literature on disciplinary literacy teaching. Review of research in education, 31(1): 144.

NATALE, L. y STAGNARO, D. (2013). Desarrollo de habilidades de lectura y escritura en la trayectoria académica del ingeniero: la experiencia de un programa desafiante e innovador. Revista Argentina de Enseñanza de la Ingeniería, 2(3): 45-52.

NAVARRO, F. (2019). Aportes para una didáctica de la escritura académica basada en géneros discursivos, DELTA: Documentação de Estudos em Lingüística Teórica e Aplicada, 35(2): 1-32.

MOYANO, E. (2007). Enseñanza de habilidades discursivas en español en contexto preuniversitario: una aproximación desde la LSF. Revista Signos, 40(65) 573-608.

VÁZQUEZ, A. (2007). Consignas de escritura: Entre la palabra del docente y los significados de los estudiantes. Colección de Cuadernillos de actualización para pensar la Enseñanza Universitaria, 2(7): 1-20. 